\title{
Leucopenia y neutropenia de rápida evolución inducidas por olanzapina.
}

Leucopenia and neutropenia rapidly evolving induced by olanzapine.

\begin{abstract}
Jordi León Caballero a, Luis González Contreras b, Maria Llobet Farré c , Sonia Ramos Perdigues $^{\mathrm{d}}$, Antoni Bulbena Vilarrasa ${ }^{\mathrm{e}}$.

${ }^{a}$ Institut de Neuropsiquiatria i Addiccions. Parc de Salut Mar de Barcelona. Barcelona, España. ${ }^{b}$ Institut de Neuropsiquiatria i Addiccions. Parc de Salut Mar de Barcelona. Barcelona, España. 'Institut de Neuropsiquiatria i Addiccions. Parc de Salut Mar de Barcelona. Barcelona, España. IInstitut de Neuropsiquiatria $i$ Addiccions. Parc de Salut Mar de Barcelona. Barcelona, España. ${ }^{e}$ Institut de Neuropsiquiatria $i$ Addiccions. Parc de Salut Mar de Barcelona. Barcelona, España.
\end{abstract}

Correspondencia: Jordi León Caballero (60499@parcdesalutmar.cat)

Recibido: 06/11/2012; aceptado con modificaciones: 24/03/2013

\begin{abstract}
RESUMEN: Algunos antipsicóticos se asocian a discrasias sanguíneas. El psicofármaco que más produce agranulocitosis es la clozapina $(0,5-2 \%$ de los pacientes). La olanzapina es un antipsicótico de segunda generación con estructura química similar a la clozapina que tiene un riesgo de leucopenia/neutropenia de 1/10.000 pacientes tratados. Presentamos el caso de un paciente de 32 años sin antecedentes somáticos ni psiquiátricos, hospitalizado por un primer episodio psicótico. En la analítica previa al ingreso no había alteraciones en el hemograma (leucocitos totales $8,92 \times 10^{3} / \mathrm{ul}$, neutrófilos totales $6,99 \times 10^{3} / \mathrm{ul}$ ). A los tres días de haber iniciado tratamiento con olanzapina $20 \mathrm{mg}$ /día el recuento leucocitario había disminuido a $2,46 \times 10^{3} / \mathrm{ul}$ (neutrófilos totales $0,64 \times 10^{3 /}$ ul). Tras sustituir la olanzapina, inicialmente por risperidona y posteriormente por zuclopentixol intramuscular, el recuento leucocitario fue aumentando progresivamente. A los doce días de la retirada, el hemograma se había normalizado (leucocitos totales $5,73 \times 10^{3} / \mathrm{ul}$ ).
\end{abstract}

PALABRAS CLAVE: Olanzapina; leucopenia; neutropenia; agranulocitosis.
ABSTRACT: Some antipsychotics drugs are associated with blood dyscrasias. The psychotropic medication most frequently associated with agranulocytosis is clozapine (0,5-2\% of patients). Olanzapine is a second-generation antipsychotic with a chemical structure similar to clozapine, with a risk of neutropenic reactions of $1 / 10.000$ treated patients. We report the case of a 32-yearold man without medical or psychiatric records, who was admitted due to a first psychotic episode. In a blood test previous to hospitalization, complete blood cell count was normal (white blood cell count $8,92 \times 10^{3} / \mathrm{ul}$, neutrophilic count $6,99 \times 10^{3} / \mathrm{ul}$ ). Three days after initiation of olanzapine $20 \mathrm{mg} / \mathrm{day}$, WBC count had fallen to $2,46 \times 10^{3} / \mathrm{ul}$ (neutrophilic count $\left.0,64 \times 10^{3} / \mathrm{ul}\right)$. After replacing olanzapine, initially for risperidone and later for intramuscular zuclopentixol, WBC count gradually increased. On the twelfth day of olanzapine withdrawal, complete blood cell count had normalized (WBC count $\left.5,73 \times 10^{3} / \mathrm{ul}\right)$.

KEY WORDS: Olanzapine; leucopenia; neutropenia; agranulocytosis. 


\section{Introducción}

Está bien establecida la asociación entre discrasias sanguíneas y algunos fármacos antipsicóticos. El psicofármaco que más frecuentemente se asocia a agranulocitosis es la clozapina, con un riesgo que oscila entre el $0.5 \%$ y el $2 \%$ de los pacientes tratados (1). Los antipsicóticos de segunda generación, posteriores a la clozapina, se presentaron como una alternativa segura a ésta en cuanto a los efectos adversos hematológicos. La olanzapina tiene una estructura química similar a la clozapina, y en los estudios previos a su comercialización no se describieron discrasias sanguíneas (2). Desde su comercialización en 1996 hasta el día de hoy se han publicado aproximadamente 46 artículos que describen casos de leucopenia y agranulocitosis inducidas por olanzapina (3). El riesgo de desarrollar leucopenia/neutropenia con olanzapina es de 1/10.000 pacientes tratados (1) y se ha establecido que es el tercer antipsicótico con mayor incidencia de neutropenia (4).

Nosotros presentamos el caso de un paciente que nunca había recibido medicación antipsicótica que desarrolló leucopenia y neutropenia al poco tiempo de iniciar un tratamiento con olanzapina.

\section{Presentación de un caso}

T.A. es un varón de 32 años, natural de Pakistán, sin antecedentes psiquiátricos ni somáticos previos, que fue llevado a urgencias de nuestro hospital por alteraciones conductuales en la vía pública. Antes de ser derivado a urgencias de psiquiatría se le tomaron las constantes vitales, objetivándose una temperatura axilar de $38^{\circ} \mathrm{C}$. Se solicitó una analítica en la que únicamente destacaba Creatina Cinasa (CK) 1.125 UI/I, con un recuento leucocitario total (RLT) de $8,92 \times 10^{3} /$ ul y neutrófilos totales (NT) de $6,99 \times 10^{3} /$ ul. Se practicó también una radiografía de tórax que fue normal, por lo que el paciente fue derivado a psiquiatría con el diagnóstico de infección respiratoria de vías altas de etiología viral. Se instauró tratamiento con paracetamol 1 gr/8h y se recomendó repetir la analítica para monitorizar los niveles de CK.

En la valoración psiquiátrica se detectó la presencia de sintomatología psicótica positiva, en forma de ideación delirante de perjuicio, autorreferencialidad y alucinaciones auditivas. Estas vivencias delirantes habían sido el origen de las alteraciones conductuales y generaban gran repercusión emocional en el paciente. Se decidió realizar ingreso hospitalario en unidad de agudos de psiquiatría y se inició tratamiento con olanzapina a dosis de $20 \mathrm{mg} /$ día.

Se solicitó una nueva analítica de control a los tres días del ingreso. Los valores de CK habían descendido a 943 UI/I y en el hemograma se encontró un RLT de 
NOTAS CLÍNICAS

$2,46 \times 10^{3} / \mathrm{ul}$ con NT de $0,64 \times 10^{3} / \mathrm{ul}$. En ese momento el paciente estaba recibiendo como tratamiento olanzapina y paracetamol, iniciados ambos fármacos el primer día de ingreso. Tras revisar la literatura se asoció esta leucopenia y neutropenia con la olanzapina. Se suspendió de forma inmediata la olanzapina y se inició tratamiento con risperidona a dosis de $6 \mathrm{mg} / \mathrm{día}$, manteniéndose el tratamiento con paracetamol. Se realizó monitorización estrecha del recuento de glóbulos blancos. A los cuatro días de haber suspendido el tratamiento con olanzapina el RLT había ascendido hasta $3,66 \times 10^{3} / \mathrm{ul}$ y $\operatorname{los} \mathrm{NT}$ a $1,02 \times 10^{3} / \mathrm{ul}$, continuando los valores de CK en progresión descendente $(237 \mathrm{UI} / \mathrm{I})$.

Progresivamente, el paciente fue presentando una atenuación de la clínica psicótica y un distanciamiento emocional de las vivencias delirantes, realizando de forma gradual crítica de la clínica psicótica presente al ingreso. Debido a que el paciente poseía escasa consciencia de enfermedad se decidió iniciar tratamiento con zuclopentixol decanoato intramuscular (IM) $200 \mathrm{mg}$ cada tres semanas concomitantemente con la risperidona oral $6 \mathrm{mg} / \mathrm{día}$.

A los doce días de haber retirado la olanzapina, y a los seis días de haber iniciado el tratamiento con zuclopentixol decanoato IM, tanto el RLT como los NT se habían normalizado $\left(5,73 \times 10^{3} / \mathrm{ul}\right.$ y $2,63 \times 10^{3} / \mathrm{ul}$ respectivamente), encontrándose también los valores de CK dentro de la normalidad (63UI/I).

Ante la estabilidad clínica del paciente, se fue disminuyendo de forma paulatina el tratamiento con risperidona, y al alta recibió únicamente tratamiento con zuclopentixol decanoato IM.

\section{Discusión}

Este caso representa un claro ejemplo de reacción adversa medicamentosa infrecuente pero potencialmente muy grave. La relación etiológica entre la olanzapina y la leucopenia en el caso presentado parece definitiva por cuatro razones: 1) Ausencia de enfermedad médica concurrente que pueda explicar la leucopenia; 2) Ausencia de otro tratamiento médico potencialmente asociado con leucopenia; 3) Relación temporal entre el inicio del tratamiento y la leucopenia; 4) Remisión de la leucopenia después de suspender la olanzapina.

El mecanismo por el cual la olanzapina induce leucopenia y neutropenia sigue aún sin conocerse (5). Una de las hipótesis establecidas es que los metabolitos de la olanzapina podrían ser tóxicos para los neutrófilos y sus células madre predecesoras (6). Otra de las hipótesis más aceptadas sostiene que la olanzapina altera los niveles de moduladores endógenos de la hematopoyesis, como el GCSF (7). 
En los casos reportados en la literatura la rápida normalización del RLT y de los NT tras la suspensión de la olanzapina es la norma. Se han descrito también casos de toxicidad hematológica autolimitada, normalizándose la cifra de leucocitos aun manteniendo el tratamiento con olanzapina (8).

A pesar de que se trata de un efecto adverso poco común, creemos que es importante que los clínicos lo tengan presente dada la gravedad del mismo. Debido a que la fiebre puede ser el único signo de infección en un paciente con neutropenia debería realizarse una exhaustiva investigación en todo paciente que presente fiebre después de haber iniciado tratamiento con un antipsicótico atípico. Aunque no existen guías clínicas referentes a la monitorización del recuento de glóbulos blancos durante la administración de olanzapina creemos que es recomendable realizar un control del recuento sanguíneo completo cuando se inicia un tratamiento con este fármaco.

\section{BIBLIOGRAFÍA:}

(1) Flanagan R, Dunk L. Haematological toxicity of drugs used in psychiatry. Hum Psychopharmacol Clin Exp. 2008;23:27-41.

(2) Duggan L, Fenton M, Rathbone J, Dardennes R, El-Dosoky A, Indran S. Olanzapina para la esquizofrenia (Revisión Cochrane traducida). En: La Biblioteca Cochrane Plus, 2008 Número 2. Oxford: Update Software Ltd. Disponible en: http://www.update-software.com. (Traducida de The Cochrane Library, 2008 Issue 2. Chichester, UK: John Wiley \& Sons, Ltd.).

(3) Freedman JL, Ryan CA, Coffey BJ. Olanzapine-Induced Agranulocytosis in an Adolescent Male with Psychosis. J Child Adolesc Psychopharmacol. 2011; 21(2):185-9.

(4) Duggal HS, Singh I. Psychotropic drug-induced neutropenia. Drugs Today. 2005;41:517526.

(5) Duggal HS, Gates C, Pathak P. Olanzapine-induced neutropenia: Mechanism and treatment. J Clin Psychopharmacol. 2004;24:234-235.

(6) Konakanchi R, Grace JJ, Szarowicz R, Pato M. Olanzapine prolongation of granulocytopenia after clozapine discontinuation. J Clin Psychopharmacol. 2000;20:703-704.

(7) Schuld A, Kraus T, Hinze-Selch D, Haack M, Pollmächer T. Granulocyte colony stimulating factor plasma levels during clozapine and olanzapine induced granulocytopenia. Acta Psychiatr Scand. 2000;102:153-155.

(8) Tolosa-Vilella C, Ruiz-Ripoll A, Mari-Alfonso B, Naval-Sendra E. Olanzapine-induced agranulocytosis. A case report and review of the literature.Prog Neuro-Psychopharmacol Biol Psychiatry. 2002;26:411-417. 